\title{
Extended use of the Ariel Core Survey Data
}

\author{
C. A. Haswell ${ }^{1}$
}

Received: 31 July 2020 / Accepted: 4 November 2021 /Published online: 22 December 2021

(c) The Author(s) 2021

\begin{abstract}
The Ariel mission will execute an ambitious survey to measure transit and / or secondary eclipse spectra of the atmospheres of about 1000 exoplanets. I outline here some possible scientific applications of the exquisite Ariel Core Survey data, beyond the science for which they are primarily designed.
\end{abstract}

Keywords Stars $\cdot$ Planets $\cdot$ Photometry $\cdot$ Spectroscopy

\section{Introduction}

Along with thermal IR spectroscopy, Ariel will produce exquisite optical and nearIR photometric data, with multi-band photometry at $1 \mathrm{~Hz}$ sampling. For a generic target the total noise in each of VISPhot, FGS1 and FGS2 instruments is less than $50 \mathrm{ppm} \sqrt{ } \mathrm{hr}[39,49]$. With photometric data of this quality, Ariel is sensitive to the intrinsic variability of even very quiet stars. This short paper outlines a variety of science which might be executed with the ground-breaking data included in Ariel's Core Survey. A very short summary of some of these potential extended use applications of the Ariel Core Survey data is included in Tinetti et al. [49]; further examples and more detailed discussions can also be found in Szabó et al. [46], Borsato et al. [7], and Garai (2020).

This paper extends the work of Szabó et al. [46], Borsato et al. [7], and Garai [18] and focusses on some straightforward applications to stellar astrophysics along with some more speculative potential applications. Section 2 focusses on advances that can be made through the application of Ariel's Core Survey data to stellar astrophysics. Since almost everything we can infer about a transiting exoplanet is derived relative to the properties of the host star, these gains will indirectly advance exoplanetary science as is obvious in the material discussed in Section 2.1. Section 3 discusses serendipitous discoveries about planets and other orbiting bodies which might be anticipated in the Ariel Core survey data. The science discussed herein is

C. A. Haswell

Carole.Haswell@open.ac.uk

1 School of Physical Sciences, The Open University, Walton Hall, Milton Keynes MK7 6AA, UK 
outside the core Ariel science case, and includes some speculative applications. The paper concludes with a short summary in Section 4.

\section{Applications of Ariel Core Survey Data in stellar astrophysics}

The overwhelming majority of the photons collected by Ariel will be emitted from the host stars without any measurable influence from the exoplanets which Ariel aims to study. Consequently, it is unsurprising that the Ariel Core Survey data has significant potential to drive progress in our understanding of the outer layers of stars. We summarise here a few of the areas where we might anticipate such progress.

\subsection{Refining the radii of PLATO terrestrial exoplanet discoveries}

In high quality photometry of transits of giant planets, the shape of ingress and egress constrains the impact parameter, $b$ (see e.g. [19]). Conversely, for small planets ingress and egress occur quickly irrespective of $b$. Morris et al. [37] show that consequently PLATO's terrestrial planet radius determinations may be generally limited to around $3.6 \%$ precision. This corresponds to an $11 \%$ uncertainty in planet volume and hence bulk density. Furthermore, this does not include contributions to the uncertainty budget arising from instrumental effects or correlated noise due to stellar super- and mesogranulation. The $3.6 \%$ limit on planetary radius precision arises from stellar oscillations and granulation coupled with degeneracies between planet radius, $R_{\mathrm{P}}$, and $b$, arising from limb darkening effects. This uncertainty floor was derived using Solar data near the Solar Minimum, and can thus be considered a firm lower limit on the noise floor attainable for PLATO radius determinations for a true Earth analogue. The corresponding directly measured quantity $\mathrm{D}^{1 / 2}$, where $\mathrm{D}$ is transit depth, has $0.73 \%$ precision.

Ariel's precise multicolour photometry, particularly the longer wavelength bands provided by FGS2 and NIRSpec spectrophotometry, can lift the degeneracy between $R_{\mathrm{P}}$ and $b$. This is because (i) limb darkening is less important at longer wavelengths (see e.g. [19]), and (ii) simultaneous fits in multiple bands ameliorates the degeneracy between impact parameter and limb darkening. Ariel transit observations of PLATO small planet discoveries can straightforwardly accomplish this directly for any individual planets observed.

\subsection{Limb-darkening coefficients as a function of stellar parameters}

Ariel may further contribute more generally through well-constrained empirical limb-darkening coefficients for host stars of the full Ariel planet transit survey (cf. Dravins et al. [15], Szábo et al. [46]). These may allow limb darkening coefficients to be more accurately assigned by matching stellar characteristics. Limb-darkening coefficients can be calculated $a b$ intio from stellar atmosphere models, for example Claret [11] performs this work for the Transiting Exoplanet Survey Satellite (TESS) 
pass band. Claret [11] give coefficients for a multi-dimensional grid of models spanning the range of effective temperatures, gravities, metallicities anticipated in the TESS sample of 200,000 stars. The confrontation of the limb darkening coefficients predicted by the model atmosphere calculations with the TESS empirical light curves will provide tests of the model atmospheres. It is clear, however, that Ariel's multicolour data will effectively fix the impact parameter and lead to a new level of stringency in these tests.

\subsection{Stellar variability across the Herzsprung-Russell diagram}

At the precision of Ariel photometry, Sun-like stars are variable even at intervals with little magnetic activity. The Helioseismic and Magnetic Imager (HMI) instrument aboard the Solar Dynamics Observatory (SDO) produces full disc optical continuum intensity images and Stokes parameters for the Solar photosphere at $45 \mathrm{~s}$ time resolution [45]. These light curves were used to produce high precision discintegrated light curves of the Solar optical continuum by Morris et al. [37]. These data show a $\sim 100$ ppm variability even for a day of low magnetic activity close to the Solar Minimum (see Fig. 5 of [37]). This variability arises from $p$-mode oscillations, and stochastic variability arising from the basal level of magnetic activity which is present even in the quiet Sun, and temporal fluctuations in the convective granulation. This variability is much larger than the measurement errors on the Solar continuum intensity measured by HMI.

While Ariel's photometric precision will not approach that of the HMI Solar continuum data, Ariel's out of transit observations will produce multi-band photometric data capable of detecting the analogous variability for bright exoplanet host stars. Coadding VISphot, FGS1, and FGS2 data in 30 min bins, Ariel can achieve optical intensity light curves with $\sim 35 \mathrm{ppm}$ standard deviation for typical bright targets (cf. [49], Szábo et al. [46]). Numerical simulations suggest that the sizes of stellar photospheric granules scale inversely with stellar surface gravity. The granule size, and the granulation-induced stellar variability is thus expected to be largest for evolved stars [50]. Ariel's diverse sample of planetary systems will allow this to be directly assessed for stars across the Hertzsprung-Russell (HR) diagram.

The interplay between convection and pulsation is complex, and has been comprehensively explored from analytical (e.g. Houdek and Dupret [24]) and numerical [50] points of view. PLATO will perform long baseline asteroseismology with exquisite frequency resolution, but Ariel will complement this with high quality, high precision multi-band light curves with short baseline and high cadence. For strictly periodic phenomena such as transits and asteroseismic oscillations, PLATO's light curves will be unrivalled, but Ariel has the collecting area needed to directly reveal stochastic intensity variations in quiet stars. Intensity variations due to lowamplitude granulation and magnetic temporal variability have only been observed in this detail so far for the Sun. Kepler light curves generally only approach a standard deviation of $100 \mathrm{ppm}$ for the brightest targets and $30 \mathrm{~min}$ exposures [25]. At Ariel's precision it may be possible to assess whether the presence of very short-period planets excite stellar pulsations. There is circumstantial evidence this may happen: 
the hot Saturn DMPP-2 orbits a $\gamma$ Doradus pulsator, but the star sits in a region of the Hertzsprung-Russell where only $2 \%$ of objects pulsate [20]. Perhaps this subset are all host stars of hot planets. Ariel's precise multiband data may reveal the excitation of stellar pulsations by close-orbiting planets.

\subsection{Stellar activity and stellar flares}

Stellar activity is a key issue which must be dealt with in the extraction of Ariel's spectra of planetary atmospheres. The strategies for this are discussed in Tinetti et al. [49], here we outline the benefits of Ariel observations to scientific issues connected with stellar activity. From this perspective, transit spectroscopy is a powerful new technique for probing stellar surface inhomogeneities, i.e. effectively spatially resolving other stars $[2,15,19,27]$.

The Inouye Solar Telescope will provide high spatial and spectral resolution visible and near-IR spectropolarimetry of structures on the Sun, with wavelength coverage to $5 \mu \mathrm{m}$ with first generation instruments [14, 51]. These data will provide spectra, covering most or all of the Ariel wavelength range, of features including granulation, prominences, flares on a variety of scales, spicules, faculae, and star spots. This library of spatially resolved Solar spectra can be used as a basis for interpreting the stellar surface inhomogeneities revealed by Ariel's core survey observations of exoplanet transits. Currently we simply do not know the structural details of inhomogeneities on the surfaces of stars other than the Sun, nor can we generally measure their spatially-resolved emission spectra. Where direct measurements of stellar surface features have been made, for example for magnetically active $M$ dwarfs, there are generally significant differences in comparison to the Sun (e.g. [3, 4, 44]). The active K4 V planet host HAT-P-11, however, has a starspot distribution in latitude similar to the Sun's, though with some spots bigger than the largest seen on the Sun [36]. MHD modelling can make predictions for different chemical compositions and stellar parameters (e.g. $[6,50])$ and these models are already used to predict, assess and ameliorate the effects of stellar surface features on radial velocities ([10]; see also [5]). Cegla et al. [9] point out that photometry is a key diagnostic, so ground-based high resolution spectroscopy simultaneous with Ariel photometry is likely to produce data able to challenge and drive significant advances in modelling stellar surface magnetoconvection.

As outlined in Tinetti et al. [49] it may prove necessary to discard, for the purposes of planetary atmosphere spectroscopy, ARIEL observations which contain stellar flares. The broad-band contribution of stellar flares to the $\mathrm{K}$ band is very small, however stellar flares are inherently non-thermal phenomena, and much of their optical and IR emission is generally likely to be via Paschen, Brackett and Pfundt line emission from neutral hydrogen. Only one of these lines lies within the $\mathrm{K}$ band. In the instances where flaring activity is detected, for example in the shortest-wavelength FGS1 band, the ARIEL IR data may reveal the associated temporally varying Paschen, Brackett and Pfundt line and continuum emission. These data can be interpreted to derive detailed physical properties of the stellar atmospheres, guided by high quality spectral atlases for solar flares. 
A minority of solar flares have anomalously low hydrogen line emission fluxes. These "white light" flares (WLFs) have emission which is dominated by the freebound continuum emission from recombining hydrogen ions [42]. WLFs are attributed to significant amounts of energy being deposited deeper than usual in the solar atmosphere [38]. Recent work concludes that this deposit of energy in the lower, rather than the upper, chromosphere can be explained more readily if the flare's energy is carried by protons rather than electrons. Energetic electron beams can deposit energy in the lower chromosphere, but require rather exotic parameters to do so [41]. Ariel core survey data could potentially probe analogous physics for any exoplanet host stars caught flaring during the observations, through comparison of the optical light curves with IR spectroscopy covering the Paschen, Brackett and Pfundt lines.

Ariel's core survey data suffering from stellar-flare contamination will extend our knowledge of the dynamical process operating in the chromospheres of other stars, building on e.g. Kowalski et al. [28, 29] and generalising inferences from the Sun to stars of a range of spectral types and luminosity classes. Ariel's VISphot, FGS1 and FGS2 photometers can in principle be read out at cadences of up to $20 \mathrm{~Hz}$, though the core survey data will have cadence limited at around $1 \mathrm{~Hz}$ [49]. Stellar flares are impulsive events powered by magnetic reconnection, and high precision photometry at higher cadences could open up new parameter space for discovery. Coupled with the ARIEL IR spectrophotometry and the rich contextual data from 21st Century Solar observations, Ariel Core Survey data may resolve uncertainties about the optically thin outer layers of stars across the HR diagram. Some of ARIEL's core survey targets may exhibit magnetic star-planet interactions. These can perhaps be characterised at unprecedented precision through ARIEL photometric data.

\section{Applications of Ariel Core Survey data to exoplanets and other orbiting bodies}

Ariel's core science is the study of planetary atmospheres through transmission spectroscopy during exoplanet transits, emission spectra derived from secondary eclipses, and through the acquisition of exoplanet phase curves. The Core Survey data may, however, also provide information on other aspects of the exoplanetary systems observed.

\subsection{Planet surface maps from secondary eclipses}

ARIEL can build on Spitzer's legacy of planet mapping using secondary eclipse and phase curve data. By the time Ariel is operational, there will probably be a number of rocky planets amenable to mapping; certainly the mapping of the planet LHS 3844b [30] can be repeated, and extended in wavelength coverage. Very precise high cadence light curves at eclipse ingress and egress can produce brightness maps of exoplanets [13, 34, 35]. This builds on decades of heritage of similar techniques applied to eclipsing binary systems, notably in cataclysmic 
variable stars (e.g. [40]). It may even prove possible to detect signs of plate tectonics on exoplanets. Active volcanoes on Io are bright at 2-5 $\mu$ [12]. Eclipse maps from Ariel data may indicate the outlines of rocky exoplanets' tectonic plates, just as the volcanoes on Earth trace terrestrial subduction zones [53].

\subsection{Exomoons and exorings}

Exomoons can cause both transit timing and transit duration variations through their gravitational interactions with the transiting planet they orbit [26]. As discussed in Borsato et al. [7], Ariel will make precise measurements of both. The exomoon itself can also be directly detected through its transit. The combination of these effects observed with ARIEL's photometric and timing precision will produce data both sensitive to exomoons and powerfully able to determine the mass and size of any detected. Currently the best exomoon candidate, orbiting Kepler-1625 b, may be attributed to instrumental systematics [22, 31, 47, 48]. ARIEL's suite of instruments are all capable of producing sensitive, high cadence data, and systematic effects in their light curves should be largely uncorrelated. This will help enormously in promptly resolving debates like that on Kepler-1625 b's putative moon [46].

Heller [21] gives a comprehensive review of the methods which can reveal both exomoons and exorings. In the Solar System, rings are ubiquitous around giant planets, and have even been detected around an asteroid [8]. Rings are unlikely to produce dynamical effects on transit timing, but can be directly detected through the flux dips due to their own transit across the host star [52]. The light curves of transiting ring systems might also exhibit forward-scattering (e.g. [1]). Ariel's multi-band photometric capabilities will reveal any ring systems around core survey targets, and characterise those already identified by PLATO.

\subsection{Exocomets}

Exocomets were first found orbiting $\beta$ Pic through their absorption in the Ca II H\&K lines [16]. A recent survey of 117 main sequence stars [43] found signatures of absorption from gas possibly attributable to exocomets in about $25 \%$ of the sample. The sample was biased to maximise such detections so this should not be interpreted as an occurrence rate. Nevertheless, it seems likely that Ariel's core survey data on edge-on planet-hosting stars will catch some transits of exocomets. Solar System comets contain species with plentiful spectra lines in wavelength range covered by Ariel. For example, Lippi et al. [33] shows molecular line fluorescence efficiencies within the AIRS band, scaled by typical Solar System cometary molecular abundances and terrestrial transmittance for six molecules found in Solar System comets. Clearly Ariel will not resolve the individual ro-vibrational transitions, but it seems probable that detection of the unresolved bands due to these and other cometary molecular species will be made serendipitously. 


\subsection{Exotrojans}

Trojan bodies, also known as co-orbital bodies, orbit at the L4 or L5 Lagrange points which lie respectively at points $60^{\circ}$ ahead and behind of a planet. None have yet been found in exoplanetary systems [32], though they are present in the Solar System and predicted by theories of planetary system formation [23]. Ford and Holman [17] discussed identifying exotrojans through TTVs. Ariel's photometric precision also offers prospects for direct detection through transits of $\sim 1000 \mathrm{~km}$ sized exotrojans, but the L4 and L5 points will only be covered for objects in Tier 4 (phase curves). The detection of Trojans accompanying a hot or warm giant planet would imply the planet had arrived in its present location via migration through a dissipative disc rather than via tidal circularisation of a highly eccentric orbit.

\section{Summary and conclusions}

To execute its core science goals on exoplanetary atmospheres, Ariel will amass photometry and spectrophotometry of unprecedented quality on $\sim 1000$ bright stars. These data will be a rich resource for the astrophysics of stellar photospheres and chromospheres, and will almost certainly produce serendipitous breakthroughs in our knowledge of exoplanetary systems.

Acknowledgements CAH's work on Ariel is supported by STFC under grant ST/T00178X/1.

Open Access This article is licensed under a Creative Commons Attribution 4.0 International License, which permits use, sharing, adaptation, distribution and reproduction in any medium or format, as long as you give appropriate credit to the original author(s) and the source, provide a link to the Creative Commons licence, and indicate if changes were made. The images or other third party material in this article are included in the article's Creative Commons licence, unless indicated otherwise in a credit line to the material. If material is not included in the article's Creative Commons licence and your intended use is not permitted by statutory regulation or exceeds the permitted use, you will need to obtain permission directly from the copyright holder. To view a copy of this licence, visit http://creativecommons.org/licen ses/by/4.0/.

\section{References}

1. Barnes, J.W., Fortney, J.: Transit Detectability of ring systems around extrasolar giant planets. ApJ 616, 1193 (2004)

2. Barnes, J.R., Haswell, C.A., Staab, D., Anglada-Escudé, G.: The origin of the excess transit absorption in the HD 189733 system: planet or star? MNRAS 462, 1012 (2016)

3. Barnes, J.R., Jeffers, S.V., Jones, H.R.A., Pavlenko, V., et al.: Starspot distributions on fully convective M Dwarfs: Implications for radial velocity planet searches. ApJ 812, 42 (2015)

4. Barnes, J.R., Jeffers, S.V., Haswell, C.A., Jones, H.R.A., et al.: Surprisingly different star-spot distributions on the near equal-mass equal-rotation-rate stars in the M dwarf binary GJ $65 \mathrm{AB}$. MNRAS 471, 811 (2017) 
5. Barnes, J.R., Jeffers, S.V., Anglada-Escudé, G., Haswell, C.A., et al.: Recovering planet radial velocity signals in the presence of starspot activity in fully convective stars. MNRAS 466, 1733 (2017b)

6. Beeck, B., Schüssler, M., Cameron, R.H., Reiners, A.: Three-dimensional simulations of nearsurface convection in main-sequence stars, 581, p. 42. III. The structure of small-scale magnetic flux concentrations A\&A. 581, 16 (2015). https://doi.org/10.1051/0004-6361/201525788

7. Borsato, L., Nascimbeni, V., Giampaolo, P., Szabó, Gy.: Exploiting the transit timing capabilities of ARIEL. Exp Astron (2021). https://doi.org/10.1007/s10686-021-09737-5

8. Braga-Ribas, F., Sicaedy, B., Ortiz, J.L., Snodgrass, C., et al.: A ring system detected around the Centaur (10199) Chariklo. Nature 508, 72 (2014)

9. Cegla, H.M., Watson, C.A., Shelyag, S., Mathioudakis, M., Moutari, S.: Stellar surface magnetoconvection as a source of astrophysical noise. III. Sun-as-a-star simulations and optimal noise diagnostics. ApJ 879, 55 (2019)

10. Cegla, H.M., Watson, C.A., Shelyag, S., Chaplin, W.J., et al.: Stellar Surface magneto-convection as a source of astrophysical noise. II. Center-to-limb parameterization of absorption line profiles and comparison to observations. ApJ 866, 55 (2018)

11. Claret, A.: Limb and gravity-darkening coefficients for the TESS satellite at several metallicities, surface gravities, and microturbulent velocities. A\&A 600, A30 (2017)

12. de Kleer, K., de Pater, I., Davies, A.G., Ádámkovics, M.: Near-infrared monitoring of Io and detection of a violent outburst on 29 August 2013. Icarus 242, 352 (2014)

13. de Wit, J., Gillon, M., Demory, B.-O., Seager, S.: Towards consistent mapping of distant worlds: secondary-eclipse scanning of the exoplanet HD 189733b. A\&A 548, A128 (2012)

14. Del Zanna, G., DeLuca, E.: Solar coronal lines in the visible and infrared: a rough guide. ApJ 852, 52 (2018)

15. Dravins, D., Gustavsson, G., Ludwig, H.-G.: Spatially resolved spectroscopy across stellar surfaces. III. Photospheric Fe I lines across HD 189733A (K1 V). A\&A 616, A144 (2018)

16. Ferlet, R., Hobbs, L.M., Vidal Madjar, A.: The beta Pictoris circumstellar disk. V. Time variations of the CA II-K line. A\&A 185, 267 (1987)

17. Ford, E., Holman, M.: Using transit timing observations to search for trojans of transiting extrasolar planets. ApJ Lett. 664, L51 (2007)

18. Garai, Z.: Grazing, non-transiting disintegrating exoplanets observed with the planned ARIEL space observatory. A case study using Kepler-1520b. Exp Astron (2021). https://doi.org/10.1007/ s10686-021-09750-8

19. Haswell, C.A.: 'Transiting exoplanets' Cambridge University Press, ISBN 978-0-521-19183-8 (2010)

20. Haswell, C.A., Staab, D., Barnes, J.R., et al.: Dispersed Matter Planet Project discoveries of ablating planets orbiting nearby bright stars. Nat. Astron. 4, 408 (2019)

21. Heller, R.: Detecting and characterizing exomoons and exorings. In: Handbook of Exoplanets, ISBN 978-3-319-55332-0. Springer International Publishing AG, part of Springer Nature, id.35 (2018)

22. Heller, R., Rodenbeck, K., Bruno, G.: Transit least-squares survey. I. Discovery and validation of an Earth-sized planet in the four-planet system K2-32 near the 1:2:5:7 resonance. A\&A 624, 95 (2019)

23. Horner, J., Kane, S.R., Marshall, J.P., Dalba, P.A., et al.: Solar system physics for exoplanet research PASP, in press arxiv:2004.13209 (2020)

24. Houdek, G., Dupret, M.-A.: Interaction between convection and pulsation. Living Reviews in Solar Physics, arXiv:1601.03913 (2016)

25. Howell, S.B., Ciardi, D.R., Giampapa, M.S., Everett, M.E., Silva, D.R., Szkody P.: Variability of kepler solar-like stars harboring small exoplanets. The Astronomical Journal. 151, 2 (2016)

26. Kipping, D.: Transit timing effects due to an exomoon. MNRAS 392, 181 (2009)

27. Kowalski, A., Schrijver, K., Pillet, V., Criscuoli, S.: Developing a vision for exoplanetary transit spectroscopy: a shared window on the analysis of planetary atmospheres and of stellar magnetic structure Astro2020: Decadal Survey on Astronomy and Astrophysics, science white papers, no. 149; Bull. Am. Astron. Soc. 51(3), 149 (2019)

28. Kowalski, et al.: A white light megaflare on the dM4.5e Star YZ CMi. ApJLett 714, L98 (2010)

29. Kowalski, et al.: New insights into white-light flare emission from radiative-hydrodynamic modeling of a chromospheric condensation. Sol. Phys. 290, 3487 (2015)

30. Kreidberg, L., Koll, D.B., Morley, C., Hu, R., et al.: Absence of a thick atmosphere on the terrestrial exoplanet LHS 3844b. Nature 573, 7772 (2019a) 
31. Kreidberg, L., Luger, R., Bedell, M.: No evidence for lunar transit in new analysis of hubble space telescope observations of the Kepler-1625 system. ApJLett 877, L15 (2019)

32. Leleu, A., Lillo-Box, J., Sestovic, M., Robutel, P., et al.: Co-orbital exoplanets from close-period candidates: the TOI-178 case. A\&A 624, A46 (2019)

33. Lippi, M., Villaneuva, G.L., Mumma, M.J., Carmarca, M.N., Faggi, S., Paganini, L.: New insights into the chemical composition of five oort cloud comets after re-analysis of their infrared spectra. AJ 159, 157 (2020)

34. Louden, T., Kreidberg, L.: SPIDERMAN: an open-source code to model phase curves and secondary eclipses. MNRAS 477, 2613 (2018)

35. Majeau, C., Agol, E., Cowan, N.B.: A two-dimensional infrared map of the extrasolar planet HD 189733b. ApJLett 747, L20 (2012)

36. Morris, B., Hebb, L., Davenport, J.R.A., Graeme, R., Hawley, S.L.: The starspots of HAT-P-11: evidence for a solar-like dynamo. ApJ 846, 99 (2017)

37. Morris, B.M., Bobra, M.G., Agol, E., Lee, Y.J., Hawley, S.L.: The stellar variability noise floor for transiting exoplanet photometry with PLATO. MNRAS 493, 5489 (2020)

38. Neidig, D.F.: The importance of solar white-light flares. Solar Phys. 121, 261 (1989)

39. Pascale, E.: ARIEL Performance modelling and predictions of performance. ARIEL Open Conference https://https://www.cosmos.esa.int/documents/1783156/3739393/14-1205_PascaleEnzo_Perfo rmanceModelling.pdf/1f06586c-d850-c41e-20a7-8c633219a9c8?t=1580377625919. Accessed 31 July 2020 (2020)

40. Pratt, G.W., Hassall, B.M.J., Naylor, T., Wood, J.H., Patterson, J.: Optical and ROSAT X-ray observations of the dwarf nova OY Carinae in superoutburst and quiescence. MNRAS 309, 847 (1999)

41. Procházka, O., Reid, A., Milligan, R.O., et al.: Reproducing type II white-light solar flare observations with electron and proton beam simulations. ApJ 862, 76 (2018)

42. Procházka, O.: Analysis and modelling of a type II white-light solar flare. PhD Thesis, Queen's University Belfast (2019)

43. Rebollido, I., Eiroa, C., Montesinos, B., Madonado, J., et al.: Exocomets: A spectroscopic survey. A\&A 639, A11 (2020)

44. Reiners, A.: Observations of cool-star magnetic fields. Living Rev. Sol. Phys. 9(1), 1 (2012)

45. Schou, J., et al.: Polarization calibration of the Helioseismic and Magnetic Imager (HMI) onboard the Solar Dynamics Observatory (SDO). Solar Phys 275, 229 (2012)

46. Szabó, G.M., Kálmán, S., Pribulla, T. et al. High-precision photometry with Ariel. Exp Astron (2021). https://doi.org/10.1007/s10686-021-09777-x

47. Teachey, A., Kipping, D.: Evidence for a large exomoon orbiting Kepler-1625b. Sci. Adv. 4, 1784 (2018)

48. Teachey, A., Kipping, D., Burke, C.J., Angus, R., Howard, A.W.: Loose ends for the exomoon candidate host kepler-1625b. AJ 159, 142 (2020)

49. Tinetti, G., et al.: Ariel, Atmospheric remote-sensing infrared exoplanet large-survey. Enabling planetary science across light-years. ESA Definition Study Report (2020)

50. Trampedach, R., Asplund, M., Collet, R., Nordlund, A., Stein, R.F.: A grid of three-dimensional stellar atmosphere models of solar metallicity. I. General Properties, granulation, and atmospheric expansion. ApJ 769, 18 (2013)

51. Tritschler, A., et al.: (DKIST Team): Daniel K. Inouye Solar Telescope: High-resolution observing of the dynamic. Sun Astron Nachr. 37, 1064 (2016)

52. Tusnski, L.R.M., Valio, A.: Transit model of planets with moon and ring systems. ApJ 743, 97 (2011)

53. Zanazzi, J.J., Triaud, A.H.M.J.: The ability of significant tidal stress to initiate plate tectonics. Icarus 325, 55 (2019)

Publisher's Note Springer Nature remains neutral with regard to jurisdictional claims in published maps and institutional affiliations. 